\title{
Effect of tramadol on the awake time and extubation response of elderly patients at different times of general anesthesia recovery stage
}

\author{
Hang Wu ${ }^{1, a}$, Guoqing Zhao ${ }^{1, b}$, Shuang Qi ${ }^{1, c^{*}}$ \\ ${ }^{1}$ China-Japan Union Hospital of Jilin University, Changchun130033, China \\ a1092551905@qq.com, ${ }^{\mathrm{b}} 403952434 @ q q . c o m,{ }^{\mathrm{c}}$ qishuang@jlu.edu.cn \\ *corresponding author
}

Keywords: Tramadol, elderly patients, waking time, extubation reaction

\begin{abstract}
To observe the effect of intravenous low dose tramadol on awake time and extubation response in elderly patients at different time of recovery from general anesthesia. 60 elderly patients were randomly divided into tramadol group (intravenous tramadol $0.7 \mathrm{mg} / \mathrm{kg}$ subgroups 30, 20 and 10 minutes before the end of operation, expressed as Igroup、 IIgroup and III group respectively) and the control group. The time of extubation, awake time and adverse reactions were observed, VAS visual analogue score and Robertson consciousness score were performed. The VAS visual analogue score at 45 minutes after operation in tramadol group was significantly better than that in control group ( $\mathrm{P}<0.05$ ), and the incidence of restlessness was low. The awake time was prolonged obviously in III group $(\mathrm{P}<0.05)$ with Robertson consciousness score at 5 minutes decreased after operation $(\mathrm{P}<0.05)$, and awakening delayed in 4 cases. The incidence of restlessness is higher in Igroup than the other two tramadol subgroups. Low dose tramadol can inhibit the extubation reaction during the recovery period of general anesthesia in the elderly patients. Tramadol is used at 20 minutes before the operation to wake up quickly, with less restlessness and delayed recovery. It may be the best time to use tramadol in the recovery period of general anesthesia.
\end{abstract}

\section{Introduction}

As a new central analgesic drug, tramadol is often used to prevent extubation reaction during recovery period of general anesthesia in recent years. However, the side effects of tramadol induced drowsiness can prolong the wakefulness time of elderly patients ${ }^{[1]}$. In this study, we compared the effects of $0.7 \mathrm{mg} / \mathrm{kg}$ tramadol on the awake time and extubation response of the elderly during the different time of general anesthesia recovery stage and explored the best administration time of tramadol in the recovery period of general anesthesia.

\section{Materials and Methods}

\section{1 General Data}

From January to December of 2017, 60 elderly patients accepted gastrointestinal surgery in China-Japan Union Hospital of Jilin University without neurological diseases. Those elderly patients were aged at 60-75 years old and their sex was unlimited, classified as gradeII by ASA.

\section{2 The Administration Methods}

60 patients were randomly divided into tramadol group and control group. Tramadol group was divided into three subgroups 30, 20, and 10 minutes before the operation, expressed as Igroup、 IIgroup and III group respectively. Tramadol was intravenously injected $0.7 \mathrm{mg} / \mathrm{kg}$ (German Grantaire Co., Ltd., Chinese drug J20050051). Fifteen patients in each group received intramuscular injection of scopolamine $0.3 \mathrm{mg}$ at 30 minutes before operation. Midazolam $0.1 \mathrm{mg} / \mathrm{kg}$, vecuronium 
$0.1 \mathrm{mg} / \mathrm{kg}$, propofol $1 \mathrm{mg} / \mathrm{kg}$, fentanyl $5 \mathrm{ug} / \mathrm{kg}$ were used for tracheal intubation, and isoflurane and nitrous oxide were inhaled to maintain anesthesia. Intraoperative supplemental vecuronium and propofol were discontinued after the peritoneum. Remains of routine antagonist muscle relaxants were removed after operation, and tracheal catheter was removed when patient's spontaneous respiration was restored, cough and swallowing reflexes were restored, and the call was responsive. The time from the end of operation to the removal of endotracheal tube was the time of extubation. At 45 minutes after extubation, VAS visual analogue scoring was used: 0 was painless; 10 was severe pain; < 3 was good; 3 to 6 was basically satisfactory; and $>6$ was poor. We recorded the time from operation to wakefulness. At the time of extubation and 5 minutes after extubation, Robertson's method was used to score consciousness. 0 point: no reaction; 1 points: light pinching ears responded; 2 points: calling names can open your eyes ; 3 points: light sleep and intermittent eye opening; 4 points: full awake and normal conversation. Adverse reactions such as hypotension, respiratory depression (hypoxemia), nausea and vomiting, chills, restlessness and delayed recovery $(>2 \mathrm{~h})$ were recorded.

\subsection{Statistical Treatment}

SPSS 13.0 statistical software is adopted for data analysis. The enumeration data is tested by $\chi 2$.. The measurement data is expressed by mean standard deviation ( $\bar{X} \pm S$ ). The intra-group comparison is tested by matching t; intra-group comparison is analyzed by one-way variance, $\mathrm{P}<$ 0.05 meaning significant differences.

\section{Results}

\subsection{Comparison of extubation time, wakefulness time, Robertson and VAS visual analogue scale in each group.}

There was no significant difference in extubation time. In the control group, Igroup IIgroup and III group, the wakefulness time of each group was lengthened successively. The difference between the control group and the three tramadol subgroups was significant $(\mathrm{P}<0.05)$. Robertson score after extubation: the control group、Igroup、IIgroup and III group decreased in turn, and lowest in III group. VAS visual analogue scale: there was no significant difference between the three tramadol subgroups. The difference between the control group and the three tramadol subgroups was statistically significant $(\mathrm{P}<0.05)$. ( Table 1 ).

\subsection{Comparison of adverse reactions in each group}

There was no hypotension, respiratory depression (hypoxemia), nausea and vomiting and chills in each group. The control group had the most cases of restlessness in the recovery period, and the difference was significant compared with the three tramadol subgroups $(\mathrm{P}<0.05)$. III group had delayed recovery in 4 cases $(\mathrm{P}<0.05)$, are shown in Table 2.

Table 1 Comparison of extubation time, wakefulness time, Robertson and VAS Scores in each

$$
\text { group }(\bar{X} \pm S, \mathrm{n}=15)
$$

\begin{tabular}{|c|c|c|c|c|}
\hline Groups & $\begin{array}{l}\text { Extubation } \\
\text { Time }(\min )\end{array}$ & $\begin{array}{l}\text { Wakefulness } \\
\text { Time(min) }\end{array}$ & Robertson Score & VAS Score \\
\hline Control group & $14.6 \pm 5.3$ & $24.9 \pm 5.1$ & $3.3 \pm 0.7$ & $5.5 \pm 2.2$ \\
\hline Igroup & $15.1 \pm 7.1$ & $25.2 \pm 4.9$ & $3.0 \pm 0.9$ & $3.4 \pm 2.3^{\mathrm{c}}$ \\
\hline IIgroup & $16.7 \pm 4.7$ & $25.6 \pm 6.1$ & $2.9 \pm 0.6$ & $2.2 \pm 1.8^{\mathrm{c}}$ \\
\hline III group & $16.4 \pm 6.3$ & $37.2 \pm 6.6^{\mathrm{a}}$ & $2.3 \pm 0.9^{\mathrm{b}}$ & $2.1 \pm 1.7^{\mathrm{c}}$ \\
\hline
\end{tabular}

Compared with Control group, $\mathrm{P}^{\mathrm{abc}}<0.05$; 
Table 2 Comparison of adverse reactions in each group ( $\bar{X} \pm S, \mathrm{n}=15)$

\begin{tabular}{|l|l|l|l|l|l|l|}
\hline Groups & Hypotension & Hypoxemia & $\begin{array}{l}\text { Nausea } \\
\text { vomiting }\end{array}$ & chills & restlessness & $\begin{array}{l}\text { Delayed } \\
\text { recovery }\end{array}$ \\
\hline Control group & 0 & 0 & 0 & 0 & 12 & $0^{\mathrm{e}}$ \\
\hline Igroup & 0 & 0 & 0 & 0 & $3^{\mathrm{d}}$ & $0^{\mathrm{e}}$ \\
\hline IIgroup & 0 & 0 & 0 & 0 & $1^{\mathrm{d}}$ & $0^{\mathrm{e}}$ \\
\hline III group & 0 & 0 & 0 & 0 & $0^{\mathrm{d}}$ & 4 \\
\hline
\end{tabular}

Compared with Control group, $\mathrm{P}^{\mathrm{d}}<0.05$; Compared withIII group, $\mathrm{P}^{\mathrm{e}}<0.05$

\section{Discussions}

Various organs of elderly patients are in a state of degeneration, often accompanied by circulatory system and respiratory diseases. Extubation reactions and postoperative pain can lead to hypertension, myocardial ischemia, arrhythmia, respiratory depression and other complications, which increase the risk of anesthesia. Tramadol is a synthetic, non-opioid opioid receptor agonist that exerts an analgesic effect on pain transmission by inhibiting the uptake of norepinephrine and increasing the concentration of serotonin outside neurons through weak affinity to u receptors and inhibition of neuronal synapses ${ }^{[2]}$. Tramadol does not have typical opioid adverse reactions caused by u-receptor activation. The incidence of respiratory depression, addiction and drug resistance is low. Tramadol has occasional nausea, vomiting and somnolence. It is suitable for elderly patients ${ }^{[3]}$. Tramadol is metabolized through the kidney and liver, and takes effect rapidly by intravenous infusion. The effect reaches its peak in 30 minutes, the peak period is 1-4 hours, and the duration is 3-6 hours. Intravenous injection of tramadol 30 minutes before the end of the operation to reduce extubation response is precisely based on its time-effect characteristics, so that the peak period of its efficacy is just the high incidence of adverse reactions, the effect of drugs is also before adverse reactions, thus blocking the source of noxious stimulation, effective prevention and treatment of restlessness $^{[4]}$.

However, elderly patients are often accompanied by a decline in organ function, an increase in adipose tissue, an increase in apparent distribution volume, a decrease in metabolic rate, and a slowdown in drug absorption, degradation and excretion. Therefore, the onset time, the time of action and the clearance time of tramadol may be prolonged. Therefore, appropriate early intravenous injection of tramadol is the key to reduce extubation response and quick wakefulness in elderly patients. The results showed that $0.7 \mathrm{mg} / \mathrm{kg}$ tramadol at 30, 20 and 10 minutes before operation could inhibit the extubation reaction during the recovery period of general anesthesia, and the analgesic effect was good or basically satisfactory (VAS score 0-6). The control group did not receive tramadol and could not achieve the above effect. Tramadol did not affect the time of removing the endotracheal tube. Intravenous injection of tramadol for 30 minutes and 20 minutes before the end of operation did not affect waking time. However, tramadol injection 10 minutes before the end of the operation significantly prolonged the awakening time, 4 cases of which resulted in delayed awakening. These indicate that elderly patients should be cautious when using small dose tramadol for 10 minutes before the end of operation. Consciousness recovery level should be the best at 30 minutes before the end of operation, most of them can respond normally after extubation, but some patients do not tolerate tracheal tube before extubation and cause restlessness. We administered tramadol 20 minutes before the end of the operation, enabling all patients to achieve Robertson score $(>2)$ at extubation or 5 minutes after extubation, with good analgesic effect and minimal adverse reactions. Therefore, intravenous injection of tramadol 0.7 
$\mathrm{mg} / \mathrm{kg}$ for 20 minutes before the end of the operation may be the best time for elderly patients to reduce extubation reaction and achieve rapid awakening during the recovery period of general anesthesia.

\section{References}

[1] Guoqing Z,Zhenbo S, Clinical benefits of tramadol combined with nitroglycerin to aged patients on recovery from general anesthesia. J. Journal of Jilin University (Medical Edition), 5(2005)457-458.

[2] Shiming D. Clem:submitted to Anesthetic pharmacology(2000)

[3] Qian Z,Dongsen L. Effects observation on intravenous injection of tramadol at emergence of general anesthesia in prevention of agitation. J. China Modern Medicine,23(2016)100-102.

[4] Kundra TS , Kuthiala G , A comparative study on the efficacy of dexmedetomidine and tramadol on post-spinal anesthesia shivering. J. Saudi J Anaesth, 11 (2017)112-115. 